\title{
ANALYTICAL SOLUTIONS FOR NON-LINEAR CONVERSION OF A POROUS SOLID PARTICLE IN A GAS-II. NON-ISOTHERMAL CONVERSION AND NUMERICAL VERIFICATION
}

\author{
G. BREM ${ }^{\dagger}$ and J. J. H. BROUWERS \\ Twente University of Technology, Enschede, Netherlands \\ (Received 17 April 1989; accepted for publication 21 December 1989)
}

\begin{abstract}
In Part I, analytical solutions were given for the non-linear isothermal heterogeneous conversion of a porous solid particle. Account was taken of a reaction rate of general order with respect to the gas reactant, intrinsic reaction surface area and effective pore diffusion, which change with solid conversion and external film transport. In this part, the analytical solutions are extended to non-isothermal conversion. Analytical solutions for the particle overshoot temperature due to heat of reaction are derived from the governing differential equation pertaining to conservation of energy, considering the limiting cases of small and large Thiele moduli. The solutions are used to assess the effect of interaction between chemical reaction rate and particle overshoot temperature on particle conversion. The analytical solutions are shown to compare favourably with numerical simulation results.
\end{abstract}

\section{INTRODUCTION}

In Part I of this paper, analytical solutions were given for the non-linear heterogeneous conversion of a porous solid particle reacting with a surrounding gas. Account was taken of reaction rates which are of general order with respect to gas concentration, intrinsic reaction surface area and effective pore diffusion which change as a result of the evolution of the pore structure during solid conversion, decrease in particle size and external film transport.

The solutions were obtained by distinguishing two limiting cases which were associated with the value of the Thiele modulus. For small values of the Thiele modulus, the governing non-linear partial differential equations pertaining to the mass balances of solid and gaseous reactants could be simplified and solved. The results thus obtained corresponded to conversion dominated by reaction kinetics.

For large values of the Thiele modulus the conversion process was found to be strongly influenced by internal and external processes. Conversion took place in a narrow zone near the outer surface of the particle, solutions for which could be derived by employing boundary layer theory.

Composite solutions for conversion variables, valid over the entire range of values of the Thiele modulus, were obtained by combining the two limiting solutions in a consistent manner.

In the present paper the analytical descriptions for non-linear conversion of a porous particle in a gas are extended to non-isothermal conversion. Analytical solutions for the particle overshoot temperature due to heat of conversion are derived from the governing differential equation pertaining to conservation of

+Netherlands Organization for Applied Scientific Research, PO Box 342, 7300 AH Apeldoorn, Netherlands. energy. The solutions are used to assess the effect of interaction between chemical reaction rates and particle overshoot temperature on conversion behaviour. Furthermore, a detailed comparison is made with results of numerical simulations.

\section{THE ENERGY EQUATION}

The temperature of a solid particle subject to conversion due to chemical reaction can be assessed from the energy equation. Taking account of heat accumulation, heat conduction and heat generation by reaction, one can write.

$$
\begin{array}{r}
\frac{\partial}{\partial t}\left[\rho_{S}\left(1-\varepsilon_{S}\right) c_{p} T\right]=\frac{1}{r^{2}} \frac{\partial}{\partial r}\left[\lambda_{e}\left(\varepsilon_{S}\right) r^{2} \frac{\partial T}{\partial r}\right] \\
-\frac{\partial C_{S}}{\partial t}(-\Delta H)
\end{array}
$$

where $T=$ local particle temperature, $c_{p}=$ specific heat of the particle, $\lambda_{e}=$ effective heat conductivity, $\Delta H=$ heat of reaction, $\rho_{s}=$ true particle density, $C_{s}=$ local concentration of solid reactant, $r=$ radius co-ordinate, $t==$ time, and $\varepsilon_{S}=$ local porosity of the particle.

Disregarding any ignition phenomena, the initial condition is

$$
t=0, \quad T=T_{\infty} .
$$

Boundary conditions are

$$
\begin{aligned}
& r=0, \quad \frac{\partial T}{\partial r}=0 \\
& r=r_{s}, \quad \lambda_{e} \frac{\partial T}{\partial r}=h\left(T_{s}-T_{\infty}\right)+\varepsilon_{r} \sigma\left(T_{s}^{4}-T_{\infty}^{4}\right)
\end{aligned}
$$

where $T_{\infty}=$ temperature of the ambient, $T_{s}=$ temperature at particle surface, $h=$ heat transfer coefficient in the external gas layer, $\varepsilon_{r}=$ emissivity, 
$\sigma=$ Stefan -Boltzman coefficient, and $r_{s}=$ particle radius.

The heat transfer coefficient can be calculated from empirical correlations [e.g. Ranz-Marshall equation (1952) for a convective flow and Prins (1987) for a fluidized bed]. For char combustion the emissivity of the external surface of the particle, $\varepsilon_{r}$, is near unity (Timothy et al., 1983).

The heat conductivity $\lambda$ is a function of the local porosity $\varepsilon_{\mathrm{s}}$. Using the expression for $\varepsilon_{\mathrm{s}}$ given by eq(2.10) of Part $I, \lambda$ can be written as an explicit function of the (reduced) solid concentration $S$.

In dimensionless form, the energy equation can be written as

$$
\begin{aligned}
\frac{\phi_{0}^{2}}{L e} \frac{\partial}{\partial \Theta}\left[\left(1-\varepsilon_{S}\right) \Psi\right]=\frac{1}{\xi^{2}} \frac{\partial}{\partial \xi}\left[\lambda(S) \xi^{2} \frac{\partial \Psi}{\partial \xi}\right] & \\
& -\alpha \beta_{T} \phi_{0}^{2} \frac{\partial S}{\partial \Theta}
\end{aligned}
$$

where $\Psi=T / T_{\infty}-1, \Theta=k_{s} C_{A, \infty}^{m} A_{g, 0} t / C_{S, 0}, \lambda=\lambda_{e} /$ $\lambda_{e, 0}, \xi=r / R$, and $\alpha=1-\left(1-\varepsilon_{S, 0}\right)\left(1-X_{\text {ash }}\right) \beta_{T} /$ Le. Furthermore, the Thiele modulus $\phi_{0}$ is defined as:

$$
\phi_{\mathrm{o}}=R \sqrt{a k_{s} A_{g, 0} C_{A, \infty}^{m-1} / D_{e, 0}}
$$

the Prater number $\beta_{T}$ is defined as

$$
\beta_{T}=(-\Delta H) C_{A, \infty} D_{e, 0} /\left(a \lambda_{e, 0} T_{c o}\right)
$$

and the Lewis number $L e$ is defined as

$$
L e=a \lambda_{e, 0} C_{S, 0} /\left(\rho_{S} C_{p} C_{A, \infty} D_{e, 0}\right) \text {. }
$$

The initial and boundary conditions are

$\Theta=0, \quad \Psi=0$

$\xi=0, \frac{\partial \Psi}{\partial \xi}=0$

$\xi=\xi_{s},-\lambda \frac{\partial \Psi}{\partial \xi}=B i_{h, c} \Psi+B i_{h, r}\left[(\Psi+1)^{4}-1\right]$

where $B i_{h, c}$ and $B i_{h, r}$ are the Biot numbers for convective and radiant heat transfer, respectively:

$$
B i_{h, c}=R \frac{h}{\lambda_{e, 0}} \text { and } B i_{h, r}=4 R \frac{\sigma \varepsilon_{r,} T_{\infty}^{3}}{\lambda_{e, 0}}
$$

For cases where the particle overshoot temperature is small compared to the ambient temperature, $|\Psi| \ll 1$, the second term on the right-hand side of eq. (2.11), representing radiant heat transfer, can be linearised. Boundary condition (2.11) then reduces to

$$
\xi=\xi_{s},-\lambda \frac{\partial \Psi}{\partial \xi}=B i_{h}(\Psi-1)
$$

where $B i_{h}=B i_{h, c}+B i_{h, r}$. In the subsequent analysis, attention is confined to the above boundary condition.

\section{ANALYTICAL SOLUTIONS}

Upon using the dimensionless equations pertaining to the mass balances of the gaseous and solid reactant, i.e. eqs (2.11) and (2.13) of Part $I$, to replace the second term on the right-hand side of eq. (2.5), the dimensionless energy equation becomes

$$
\begin{aligned}
\frac{\phi_{\mathrm{o}}^{2}}{L e} \frac{\partial}{\partial \Theta}\left[\left(1-\varepsilon_{S}\right) \Psi\right]= & \frac{1}{\xi^{2}} \frac{\partial}{\partial \xi}\left[\lambda(S) \xi^{2} \frac{\partial \Psi}{\partial \xi}\right] \\
& +\alpha \beta_{T} \frac{1}{\xi^{2}} \frac{\partial}{\partial \xi}\left[D(S) \xi^{2} \frac{\partial C}{\partial \xi}\right]
\end{aligned}
$$

where $C$ is the reduced concentration of the gaseous reactant, and $D$ is the reduced effective diffusivity.

It is noted that, for $\phi_{0}^{2} \ll 1$, the term on the lefthand side of eq. (3.1) is small compared to the terms on the right-hand side. Furthermore, for $\phi_{0}^{2} \gg 1$, analogous to the boundary layer behaviour of $C$ discussed in Part I of this paper, $\Psi$ will vary over a region of radial extent $\phi_{0}^{-1}$, implying that the first term on the right-hand side of eq. (3.1) is of order of magnitude $\phi_{0}^{2}|\Psi|$. As a consequence, the order of magnitude of the instationary term on the left-hand side of eq. (3.1) is not larger than $(L e)^{-1}$ compared to the first term on the right-hand side of eq. (3.1). Similarly, the instationary term on the left-hand side is not larger than ( $L e$ $\left.\alpha \beta_{T}\right)^{-1}$ compared to the second term on the righthand side. For conventional applications of conversion with heat production such as char combustion, $L e$ is typically $10^{4}$, and $\beta_{T}$ is typically $10^{-2}$. In the subsequent analysis, we disregard the instationary term in the energy equation, yielding

$$
\frac{\partial}{\partial \xi}\left[\lambda(S) \xi^{2} \frac{\partial \Psi}{\partial \xi}+\alpha \beta_{T} D(S) \xi^{2} \frac{\partial C}{\partial \xi}\right]=0 .
$$

Integrating with respect to $\xi$ and application of the boundary condition that $(\partial / \partial \xi) \Psi=(\partial / \partial \xi) C=0$ at $\xi=0$, gives

$$
\frac{\partial \Psi}{\partial \xi}=-\alpha \beta_{T} \frac{D(S)}{\lambda(S)} \frac{\partial C}{\partial \xi} .
$$

Using the boundary conditions for $\Psi$ and $C$ imposed at the external surface, i.e. eq. (2.13) of this part and eq. (2.17) of Part $I$, we obtain for the overshoot temperature $\Psi_{s}$ at the particle surface the expression

$$
\Psi_{s}=\alpha \beta_{T} \frac{B i_{m}}{B i_{h}}\left(1-C_{s}\right)
$$

where $C_{s}$ is the reduced gas concentration at the external surface of the particle.

The temperature variation over the particle itself can be determined by integrating eq. (3.3):

$$
\Delta \Psi_{p}=-\alpha \beta_{T} \int_{C_{s}}^{C} \frac{D(S)}{\lambda(S)} \mathrm{d} C
$$

while the total particle overshoot temperature is equal to the sum of the right-hand sides of eqs (3.4) and (3.5):

$$
\Psi=\Psi_{s}+\Delta \Psi_{p^{*}}
$$

To assess the right-hand sides of eqs (3.4) and (3.5) use is made of the solutions for gas and solid concentration given in Part I. For this purpose, distinction is made between the cases of small and large Thiele moduli. 
(i) Solutions asymptotically valid for $\phi_{0} \ll 1$. According to the solutions valid for $\phi_{0} \ll 1$ presented in Part I, the dimensionless concentration of the gaseous reactant is to first order equal to unity. This results in zero values for the right-hand sides of eqs (3.4) and (3.5). To assess the particle overshoot temperature for $\phi_{0} \ll 1$, higher-order descriptions for $C$ have been developed. Such descriptions can be obtained by iteration, i.e. by substrituting the zero-order solutions for $C$ and $S$ into the neglected terms of the governing equations [cf. eqs (2.11) and (2.13) of Part I] and solving these for $C$. Invoking thus obtained solution in eqs (3.4) and (3.5), one obtains, for the particle overshoot temperature:

$$
\begin{aligned}
& \Psi_{s}=\frac{1}{3} \frac{\alpha \beta_{T}}{B i_{h}} \phi_{0}^{2} A(S) \\
& \Delta \Psi_{p}=\frac{1}{6} \alpha \beta_{r} \phi_{0}^{2} \frac{A(S)}{\lambda(S)}\left(1-\xi^{2}\right)
\end{aligned}
$$

where $S$ is the inverse of the solution for the solid concentration given by eq. (3.3) of Part $I$.

(ii) Solutions asymptotically valid for $\phi_{0} \gg 1$. To evaluate the right-hand side of eq. (3.5), an equation is needed which expresses $S$ as a function of $C$. In principle, such an expression can be derived from the solutions valid for $\phi_{0} \gg 1$ given in Part I. But working out the integral on the right-hand side of eq. (3.5) using this expression is practically an impossible task. A reasonable approximation is one in which $S$ in the expressions for $\lambda$, and $D$ is replaced by its average value. According to the solutions for $\phi_{0} \gg 1, S$ varies over a small boundary layer of thickness $\phi_{0}^{-1}$ near the outer surface from a value of $S^{*}$ at the outer surface to unity in the interior. Hence, the average values for $S$ is $\frac{1}{2}\left(S^{*}+1\right)$ and

$$
\begin{aligned}
& \bar{\lambda}=\lambda\left[S=\frac{1}{2}\left(S^{*}+1\right)\right] \\
& \bar{D}=D\left[S=\frac{1}{2}\left(S^{*}+1\right)\right] .
\end{aligned}
$$

Using these approximations in eq. (3.5) we have

$$
\Psi-\Psi_{s}=\alpha \beta_{T} \frac{\bar{D}}{\bar{\lambda}}\left(C_{s}-C\right)
$$

where $C$ decays rapidly to zero over a boundary layer of thickness $\phi_{0}^{-1}$. The temperature increase over the entire boundary layer is thus given by

$$
\Delta \Psi_{p}=\alpha \beta_{T} \frac{\bar{D}}{\bar{\lambda}} C_{s} .
$$

Employing the expression for $C_{s}$ derived in Part I [cf. eq. (3.23)] one finds, from eqs (3.4) and (3.12):

$$
\begin{aligned}
\Psi_{s} & =\frac{\alpha \beta_{T} \phi_{0} u}{B i_{h}}\left(1-S^{*}\right) \\
\Delta \Psi_{p} & =\alpha \beta_{T} \frac{\bar{D}}{\overline{\bar{\lambda}}}\left[1-u \frac{\phi_{0}}{B i_{m}}\left(1-S^{*}\right)\right]
\end{aligned}
$$

where the boundary layer velocity $u$ has been specified in Part $I$ of this paper.

(iii) Composite solution. To arrive at an analytical description of the overshoot temperature which is applicable over the entire range of values of the Thiele modulus, the previously derived asymptotic solutions are combined as follows:

$$
\Psi(\xi)=\left\{\Psi_{1}^{-\delta}(\xi)+\Psi_{2}^{-\delta}(\xi)\right\}^{-1 / \delta}
$$

where $\Psi_{1}(\xi)$ is the particle overshoot temperature valid when $\phi_{0} \ll 1$, given by eqs (3.6)-(3.8), and $\Psi_{2}(\xi)$ is the particle overshoot temperature valid if $\phi_{0} \gg 1$, given by eqs (3.6), (3.13) and (3.14). Noting that $\Psi_{1}=O\left(\phi_{0}^{2}\right)$ and $\Psi_{2} \leqslant O\left(\phi_{0}\right)$, it can be verified from eq. (3.15) that, for $\delta>0, \Psi$ approaches $\Psi_{1}$ as $\phi_{0} \rightarrow 0$ and $\Psi$ approaches $\Psi_{2}$ as $\phi_{0} \rightarrow \infty$ (as it should be).

A value for the fit parameter $\delta$ can be obtained from a comparison with solutions for the linear case, i.e. $m=1, A(S)=D(S)=\lambda(S)=1, S^{*}=0$ and $\xi_{s}=1$. In this case it is possible to derive an exact solution for the distribution of the gaseous reactant inside the particle which is valid over the entire range of $\phi_{0}$ values (Froment and Bischoff, 1979). Upon substituting this solution into the right-hand side of eqs (3.4) and (3.5), one finds, for the overshoot temperature in the particle centre $(\xi=0)$, the expression

$$
\begin{aligned}
\Psi(\xi=0)_{\text {exact }}=\alpha \beta_{T}\left[\frac{B i_{m}}{B i_{h}}\right. & \left(1-C_{s}\right) \\
& \left.+C_{s}\left(1-\frac{\phi_{0}}{\sinh \phi_{0}}\right)\right]
\end{aligned}
$$

where

$$
C_{s}=\left[1+\frac{1}{B i_{m}}\left(\frac{\phi_{o}}{\tanh \phi_{0}}-1\right)\right]^{-1} .
$$

For the linear case, the present solutions reduce to eq. (3.15) with

$$
\Psi_{1}(\xi=0)=\alpha \beta_{T} \phi_{\hat{\sigma}}^{2}\left(\frac{1}{3 B i_{h}}+\frac{1}{6}\right)
$$

and

$$
\Psi_{2}(\xi=0)=\alpha \beta_{T} \frac{1+\phi_{0} / B i_{h}}{1+\phi_{0} / B i_{m}} .
$$

Noting that $\delta>0$, the asymptotic limits of the present solution can be shown to be equal (as it should be) to the asymptotic limits of the exact solution [cf. eq. (3.16)]:

$$
\Psi(\xi=0)_{\text {exact }} \sim \Psi_{1}(\xi=0) \text { for } \phi_{0} \ll 1
$$

and

$$
\Psi(\xi=0)_{\text {exact }} \sim \Psi_{2}(\xi=0) \text { for } \phi_{0} \gg 1 .
$$

In Fig. 1 plots have been presented of $\Psi(\xi=0)_{\text {exact }}$ and $\Psi(\xi=0)$ for $\delta=1,1.3,2$ and $\infty$ vs $\phi_{0}$, assuming $B i_{h}=B i_{m}=100$, and $B i_{h}=B i_{m}=1$. It is seen that $\delta=1.3$ gives good agreement with the exact solution. In Section 5 the solutions for particle overshoot temperature are compared with results of numerical simulations. Also here a value for $\delta=1.3$ is found to give good agreement.

In summary the present composite solution for particle overshoot temperature is equal to the "exact" solution for the limiting cases of $\phi_{0} \ll 1$ and $\phi_{0} \gg 1$, 


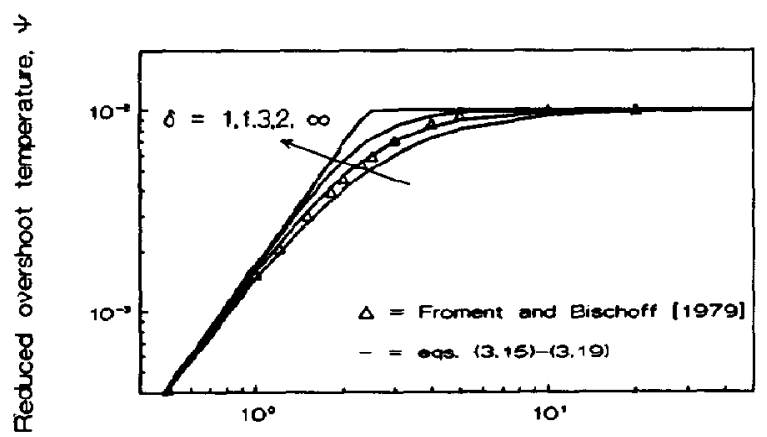

(a)

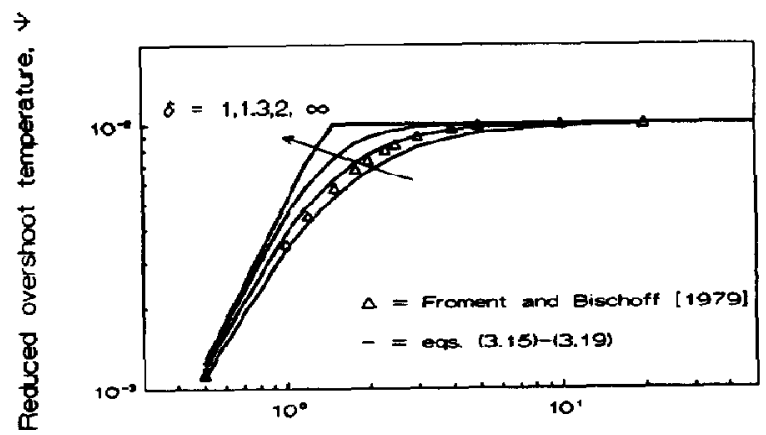

(b)

Thiele modulus, \$o

Fig. 1. Reduced overshoot temperature $\Psi$ vs Thiele number $\phi_{0}$ : comparison between exact solutions of Froment and Bischoff (1979) and the present solutions of eqs (3.15)-(3.19) valid for the linear case. (a) $B i_{h}=B i_{m}=100$ and $\alpha \beta_{T}=0.01$. (b) $B i_{h}=B i_{m}=1$ and $\alpha \beta_{T}=0.01$.

and agrees well with "exact" results in the intermediate range of $\phi_{0}$ values taking $\delta=1.3$.

\section{NON-ISOTIIERMAL BEHAVIOUR}

Interaction between particle temperature and solid conversion occurs through the reaction rate constant $k_{s}$ in the reactivity equation [cf. eqs (2.3) and (2.4) of Part 1$]$. The value of this constant is highly dependent on temperature. Increase in temperature due to heat of conversion can have a significant effect on the value of the reaction rate constant which in turn affects the conversion process and hence heat of conversion. Assessing the interaction between temperature and conversion is the purpose of this section.

Employing the Arrhenius forms, the reaction rate constant $k_{s}$ can be written as

$$
k_{s}=k_{0} \exp \left(\frac{-E_{0}}{R T}\right) .
$$

In terms of the dimensionless particle overshoot temperature $\Psi=T / T_{\infty}-1$, we have

$$
k_{s}=k_{s, 0} \exp \left[\gamma\left(\frac{\Psi}{1+\Psi}\right)\right]
$$

where $k_{s, 0}$ is the reaction rate constant based on the ambient temperature $T_{\infty}$ in eq. (4.1), and $\gamma$ is the Arrhenius number defined as $\gamma=E_{0} / R T_{\infty}$.

The exponential term in eq. (4.2) represents the influence of the particle overshoot temperature. The Arrhenius number $\gamma$ in the exponent of this equation magnifies the effect of $\Psi$. For char combustion $\gamma$ can have values between 10 and 20 . Particles overshoot temperatures of $10 \%$ of the ambient temperature then result in significant increases of the value of $k_{s}$.

Adopting eq. (4.2) in the reactivity equation results in a complete coupling between the partial differential equations pertaining to conservation of mass [cf. eqs (2.1)-(2.4) of Part I], and the partial differential equation pertaining to conservation of energy [cf. eq. (2.1)]. The solution of such coupled equations in analytical form would be quite a formidable task.

It is noted, however, that a major contribution to the particle overshoot temperature stems from the overshoot temperature at the external surface apparent when $\phi_{0} \gg 1$ [cf. eq. (3.13)]. This contribution is independent on time and place. It only leads to a parametric coupling between the mass balances equations and the energy equation, leaving the distributions with respect to time and place unaffected. All the other terms contributing to $\Psi$ [cf. eqs (3.7), (3.8) and (3.11)] vary with place and/or time. The magnitude of these terms, however, is not larger then order of magnitude $\beta_{\mathrm{T}}$. As for conventional applications such as char combustion, $\beta_{T} \ll 1$, the contribution of these terms to the value of the reaction rate constant remains in general of limited size.

In view of the above a reasonable approach to assess the effect of particle overshoot temperature on reaction rate constant is one in which the internal temperature is averaged over time of conversion and over region of conversion. For the case $\phi_{0} \ll 1$, this implies averaging the right-hand sides of eqs (3.7) and (3.8) as

$$
\begin{aligned}
\vec{\Psi}_{1} & =\frac{1}{\Theta_{c}} \int_{\Theta=0}^{\Theta_{c}} \int_{\xi=0}^{\xi_{s}}\left(\Psi_{s}+\Delta \Psi_{p}\right) \mathrm{d} \xi \mathrm{d} \Theta \\
& =\frac{1}{3} \propto \beta_{T} \phi_{0}^{2}\left(\frac{1}{B i_{h}}+\frac{1}{3}\right) \frac{X_{\infty}}{\Theta_{c}}
\end{aligned}
$$

where $\Theta_{c}$ is the time needed to reach an ultimate conversion degree of $X_{\infty}$ [see eq. (3.7) of Part I]. In eq. (4.3), $\lambda$ is assumed to equal unity, and $B i_{h}$ is assumed to be constant during conversion.

For $\phi_{0} \gg 1$, conversion takes place in a boundary layer near the external surface. The average value of the overshoot temperature in this boundary layer is approximately equal to half the overshoot temperature over the entire boundary layer given by eq. (3.14), supplemented with the overshoot temperature at the surface given by eq. (3.13), i.e.

$$
\begin{array}{r}
\bar{\Psi}_{2}=\frac{\alpha \beta_{T} \phi_{0}^{2}}{B i_{h} \Theta_{c}}\left(1-\xi_{s, \infty}\right)\left(1-S^{*}\right)+\frac{1}{2} \alpha \beta_{T} \frac{\bar{D}}{\bar{\lambda}} \\
\times\left[1-\frac{\phi_{0}^{2}}{B i_{m} \Theta_{c}}\left(1-\xi_{s, \infty}\right)\left(1-S^{*}\right)\right]
\end{array}
$$


where $\Theta_{c}$ is the conversion time needed to reach an ultimate particle diameter $\xi_{s, \infty}[$ see eq. (3.37) of Part I].

A composite solution for the average overshoot temperature valid over the entire range of $\phi_{0}$ values can be expressed as

$$
\bar{\Psi}=\left(\bar{\Psi}_{1}^{-\delta}+\bar{\Psi}_{2}^{-\delta}\right)^{-1 / \delta}
$$

where $\delta$ is taken equal to the value adopted in eq. (3.15), i.e. $\delta=1.3$.

In the dimensionless versions of the differential equations pertaining to conservation of mass and energy, only the Thiele modulus depends on the value of the reaction rate constant. A simple iterative procedure to assess the Thiele modulus under non-isothermal conditions is then as follows:

(i) Assume the particle temperature to be equal to the ambient temperature, i.e. $\bar{\Psi}=0$.

(ii) Calculate the reaction rate constant from eq. (4.2) taking $\Psi=\bar{\Psi}$ and the Thiele modulus from eq. (2.6).

(iii) Calculate the average overshoot temperature $\overline{\mathbf{Y}}$ from eqs (4.3)-(4.5) and return to (ii).

The above iteration can be continued until sufficient convergence in the values of $\phi_{0}$ and $k_{s}$ is obtained. In general a few iterations are sufficient. Thus obtained values for $\phi_{0}$ and $k_{s}$ can directly be used to determine the value of any output variable specified in Parts I and II of this paper. The resulting solutions properly take account of non-isothermal behaviour for cases where the previously mentioned assumptions are satisfied. Illustrations of non-isothermal behaviour are given in Section 5 .

It is noted that, for large $\beta_{T}$ and for a narrow range of $\phi_{o}$ values, more than one possible value of $\Psi$ could be obtained (Aris, 1975). This behaviour is caused by the fact that the heat generation term, i.e. the second term on the right-hand side eq. (2.5), is a strongly non-linear function of $\Psi$, which can lead to multiple solutions of the equations. Criteria for these events, have been derived by several investigators. Froment and Bischoff (1979), for example, presented that for first-order reactions. The proper sufficient criterion for uniqueness of the steady state is

$$
\beta_{T} \gamma<4\left(\frac{B i_{h}}{B i_{m}}+\beta_{T}\right)
$$

\section{NUMERICAL VERIFICATION OF THE ANALYTICAL SOLUTIONS}

The analytical solutions for isothermal and nonisothermal conversion have been verified using numerical simulation results. The verification involved specific expressions for the reaction surface area $A_{g}$, the effective diffusivity $D_{e}$, and the effective heat conductivity $\lambda_{e}$. For the reduced reaction surface area $A$, an expression conforming to Wen (1968) was adopted:

$$
A(S)=\frac{A_{g}}{A_{g, 0}}=S^{n}
$$

where $A_{g, 0}$ is the initial reaction surface area.

For the effective diffusivity, we adopted the expression given by Laurendeau (1978):

$$
D_{e}=c \varepsilon_{s}^{\beta}
$$

Experiments with porous particles have shown that $\beta$ can have values which vary between 2 and 3 (Satterfield and Sherwood, 1963; Bliek, 1984).

For the effective heat conductivity, the expression given by Sotirchos and Amundson (1984) has been applied:

$$
\lambda_{e}=\left(1-\varepsilon_{s}\right)^{\beta} \lambda_{e, s}+\varepsilon{ }_{s}^{\beta} \lambda_{e, A}
$$

where $\lambda_{e, s}=$ heat conductivity of the solid, and $\lambda_{e, A}=$ heat conductivity of the gas penetrated.

Applying eq. (2.10) of Part I, $D_{e}$ and $\lambda_{e}$ can be written as explieit functions of the reduced solid concentration $S$.

\subsection{Isothermal conversion}

The set of equations governing the isothermal reaction of a particle in a gas has been solved numerically using a fourth-order Runge-Kutta technique. The set of equations including the boundary conditions has been given in Section 2 of Part I of this paper, supplemented with eqs (5.1)-(5.3) above. The particle sphere was divided in a great number of shell layers, depending on the Thiele modulus. As a first step, eq. (2.11) of Part $I$ is integrated while the value of the gas concentration in the centre of the particle is varied until the gas concentration gradient at the particle surface fits boundary condition (2.17) of Part I. Subsequently, the solid concentration on the new time line is estimated using eq. (2.13) of Part I. This procedure is repeated until the ultimate conversion degree is reached. The gas mass balance is used to estimate the accuracy of the solution. The maximum error was about $3 \%$.

In the following analysis, the numerical calculations are used to verify the analytical solutions for both the regimes $\phi_{0} \ll 1$ and $\phi_{0} \gg 1$ considering a number of typical cases. Subsequently, the composite analytical solution, valid over the entire range of $\phi_{0}$ values, given in Section 4 of Part $I$, is verified.

(i) Comparison with solutions for $\phi_{0} \ll 1$. For $\phi_{0} \ll 1$, the conversion time vs conversion degree, given by eq. (3.7) of Part $I$, is compared with numerical results for different values of $n$ (see Fig. 2). It is noted that, for $n \geqslant 1$, complete conversion $\left(X_{\infty}=1\right)$ is only attainable for $\Theta \rightarrow \infty$. As shown in Fig. 3 the conversion rate vs conversion degree, given by eq. (3.6) of Part I, also compares very well with numerical results.

(ii) Comparison with solutions for $\phi_{0} \gg 1$. For $\phi_{0} \gg 1$ the ultimate conversion time vs $B i_{m}$, given by eq. (3.31) of Part $I$, is compared with numerical results for different values of $\boldsymbol{n}$ (see Fig. 4). For low values of $B i_{m}$ (conversion controlled by external film transport), 


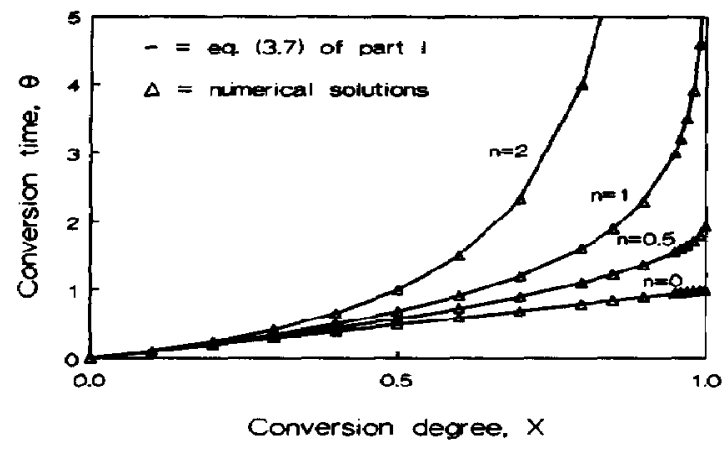

Fig. 2. Conversion time $\Theta$ as a function of the conversion degree of the solid for $\phi_{0}=0.1$, and various values of $n$.

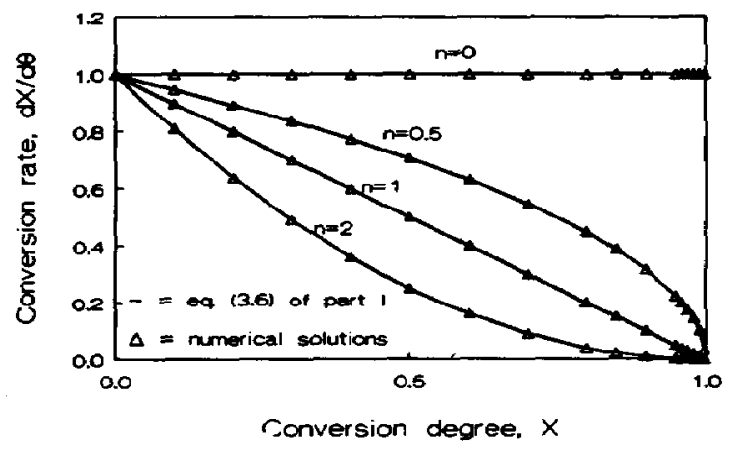

Fig. 3. Conversion rate as a function of conversion degree of the solid for $\phi_{0}=0.1$, and various values of $n$.

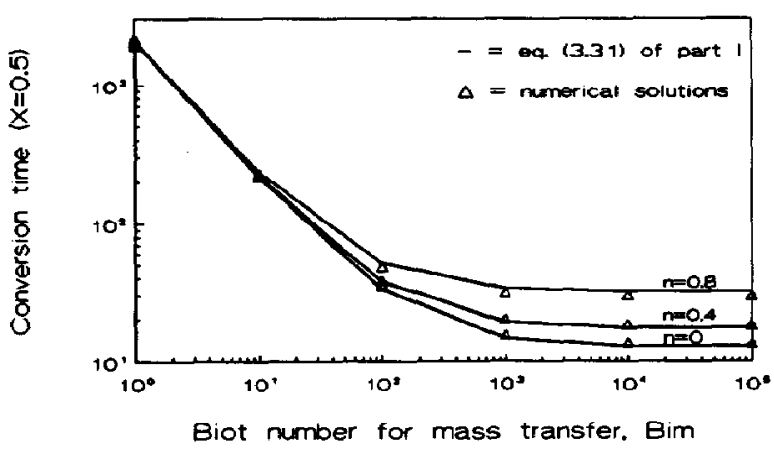

Fig. 4. Time required for $X=0.5$, as a function of $B i_{m}$ for various values of $n$ : $\phi_{0}=100, S^{*}=0, \beta=2, m=1$ and $x_{\text {ash }}=0$.

the time required for $X=0.5$ is almost independent on $n$. For large values of $B i_{m}$ (conversion controlled by pore diffusion), time for $X=0.5$ is strongly dependent on $n$, but almost independent on $B i_{m}$, as expected. It is noted that the numerical results agree very well with the present solutions.

The results of a further analysis of the pore diffusion controlled regime, i.e. $\phi_{0} \gg 1$ and $B i_{m} \gg \phi_{0}$, is given in Fig. 5. Here, the time required for $X=0.5$ is given as a function of $\beta$, which characterizes the evolution of the pore diffusion, with $n$ as a parameter which char-

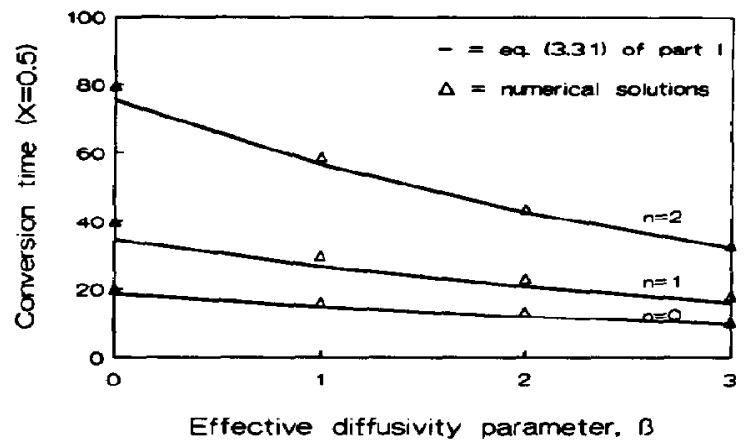

Fig. 5. Time required for $X=0.5$ as a function of $\beta$ for various values of $n: \phi_{0}=100, B i_{m}=10,000, S^{*}=0.2$, $\varepsilon_{s, o}=0.5, m=1$ and $X_{\text {ash }}=0$.

acterizes the evolution of the reaction surface area. For low $n$ values, the influence of $\beta$ diminishes, while, for high $n$ values, the time required for $X=0.5$ strongly decreases with $\beta$. It can be seen that analytical and numerical results agree quite well.

In Fig. 6 the conversion rate, given by eq. (3.30) of Part $I$, is shown as a function of the conversion degree $X$ for $\phi_{0}=100$ and and various values of $B i_{m}$. In all cases the results show a good agreement between numerical solutions and the present analytical solutions.

(iii) Comparison with composite solutions. Figure 7 shows the results of a numerical verification of the composite solution for the conversion time given in eq. (4.1) of Part $I$ for the entire range of $\phi_{0}$ values while $B i_{m}=100$ and 1 . The composite solution is given for different values of $\kappa$. It is noted that $\kappa$ equal to 2 gives the best agreement between the numerical solution and the present composite solution. In Fig. 8 the results are shown of a numerical verification of the composite solution for the conversion rate given in eq. (4.2) of Part I vs the conversion degree taking an intermediate value for the Thiele modulus: $\phi_{0}=5$. It is seen that the numerical solution shows an initial increase of the conversion rate caused by the transient behaviour which is omitted in the present solutions.

In Fig. 9 results are shown of a numerical verification of the composite solution with $\kappa=2$ for various values of the reaction order $m$ in the gaseous reactant. It can be seen that for th chosen value of the Thiele modulus $\phi_{0}=3$, the maximum difference between the numerical solution and the composite solution for the conversion time $\Theta(X=0.5)$ is only $10 \%$.

Finally, Fig. 10 shows the influence of the nonlinear parameters $m, n, \beta$ and $S^{*}$ on the complete conversion time. It can be seen that, if $m$, which is the reaction order in gas concentration, decreases, conversion time decreases because of increasing reactivity. If $n$, which characterizes the evolution of the reaction surface area, increases, conversion time increases because of decreasing reactivity. If $\beta$, which characterizes the evolution of the pore diffusion, increases, conversion time decreases because of increasing effective diffusivity. If $S^{*}$, which is the critical solid 


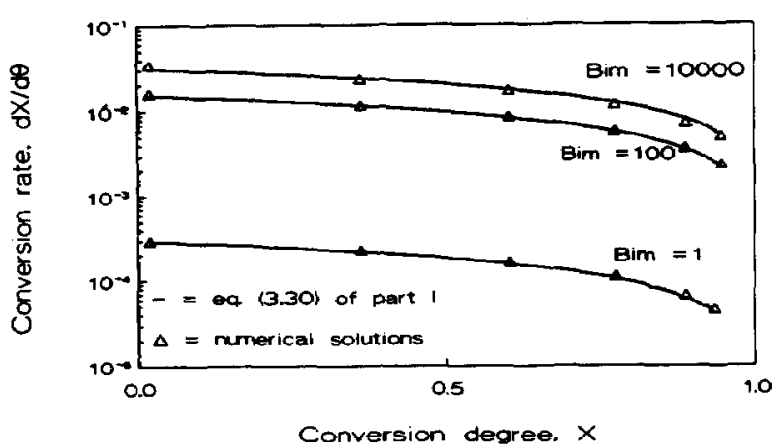

Fig. 0 . Conversion rate as a function of the conversion degree of the solid for $\phi_{0}=100$ and $B i_{m}=1,100$ and 10,000 : $n=0.5, m=1, S^{*}=0, X_{\text {ash }}=0, \beta=2$ and $\varepsilon_{s, 0}=0.5$.
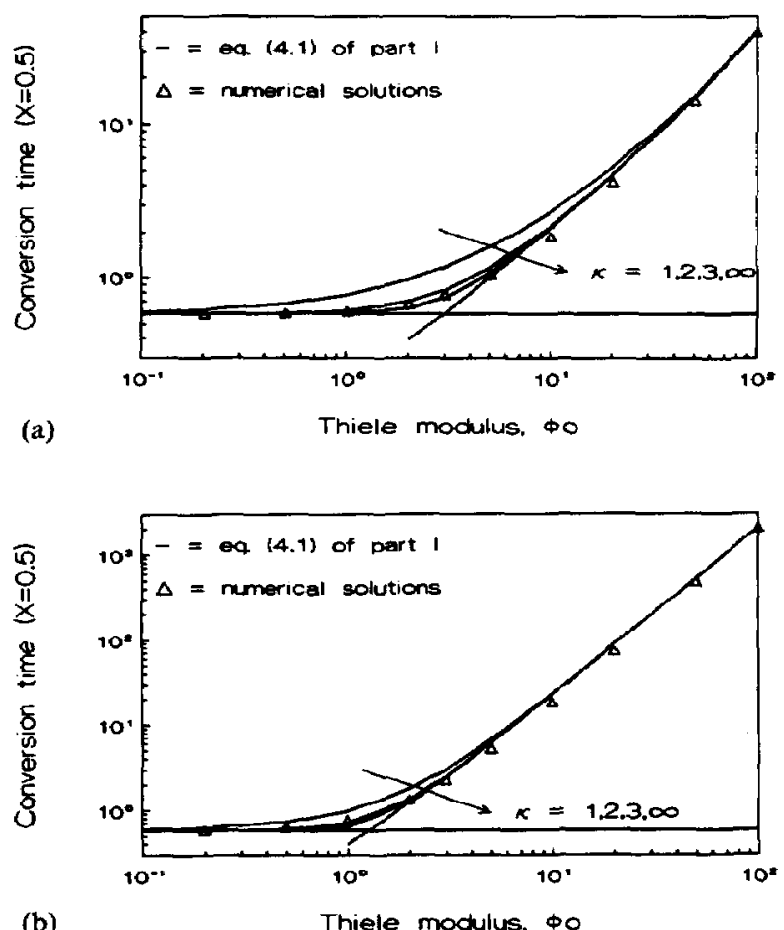

(b)

Fig. 7. Results of a numerical verification of the composite solution of the conversion time in eq. (4.1) of Part I for the entire range of $\phi_{0}$ values for $B i_{m}=100(a)$ and $B i_{m}=1$ (b):

$$
n=0.5, m=1, S^{*}=0, X_{\text {ash }}=0, \varepsilon_{s .0}=0 \text { and } \beta=2 \text {. }
$$

concentration at the outer surface of the particle, increases, conversion time decreases, but on the other hand solid loss will increase. Because of the great sensitivity of the conversion time for the mentioned non-linear parameters, an accurate estimation of the values of these parameters is required to assess real conversion behaviour.

In summary, it is concluded that the analytical solutions valid for $\phi_{0} \ll 1$ and $\phi_{0} \gg 1$ compare favourably with numerical solution results of corresponding cases. Analytical and numerical results agree quite well for intermediate values of $\phi_{0}$.

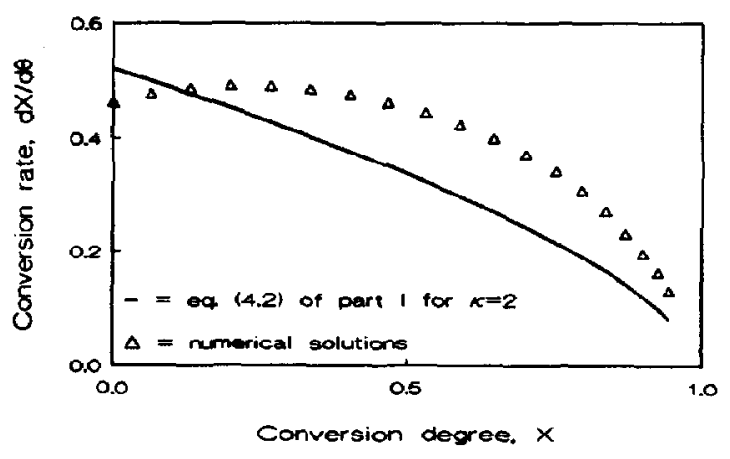

Fig. 8. Results of a numerical verification of the composite solution for the conversion rate of eq. (4.2) of Part $I: \phi_{0}=5$, $B i_{m}=100, n=0.5, m=1, \quad \beta=2, S^{*}=0, \quad X_{\text {ash }}=0$ and $\varepsilon_{\mathrm{S}, 0}=0.5$.

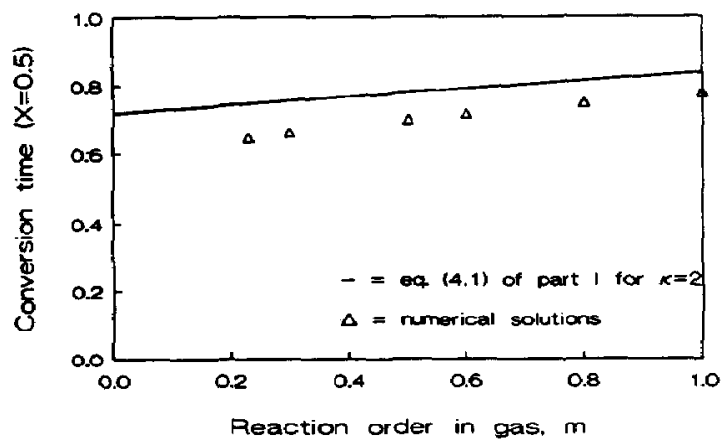

Fig. 9. Conversion time as a function of the reaction order $m$ : $\phi_{0}=3, B i_{m}=100, n=0.5, \beta=2, S^{*}=0, X_{\text {ash }}=0$ and $\varepsilon_{s, 0}=05$.

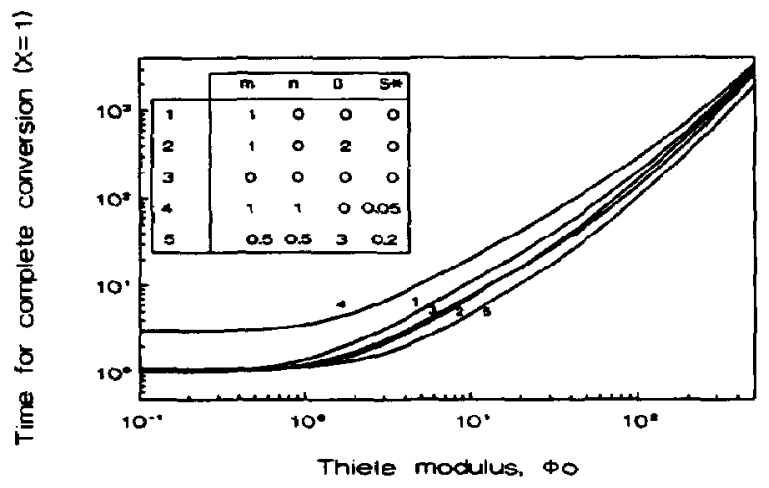

Fig. 10. Time for complete conversion as a function of the Thiele modulus for various values of the non-linear parameters.

\subsection{Non-isothermal conversion}

To verify the present analytical solutions, for nonisothermal conversion, use has been made of a numer ical model, the ASPC model, developed by Prins (1987). The ASPC model is based on the dusty gas mass flux equations for mass transport through porous media, and allows computations of concentrations and mole fluxes of the various components $(\mathrm{CO}$; 
$\mathrm{CO}_{2}, \mathrm{O}_{2}$ and $\mathrm{N}_{2}$ ) involved in the combustion reaction as a function of the radial position inside the particle.

The main assumptions within the ASPC model, with respect to the present solutions, are $m=1, n=1$, $\beta=3, \varepsilon^{*}=0.95, X_{\text {ash }}=0$ and $\varepsilon_{S, a}=0$.

Prins used the ASPC model to investigate the combustion rate of carbon particles in a fluidized bed. For the numerical verification the value of the main parameters were:

- the kinetic constant $k_{s}$ is $1.3 E 7$ exp $(-20,000 /$ T) $\mathrm{kg} /\left(\mathrm{kg} \mathrm{s} \mathrm{kg} \mathrm{O} / \mathrm{m}^{3}\right)$;

- the mass transfer coefficient $k_{d}$ and the heat transfer coefficient $h$, according to the relations given by Prins (1987);

- heat transfer by radiation is neglected $\left(\varepsilon_{r}=0\right)$;

- the burn-out time is defined for $X_{\infty}=0.9$;

- the initial porosity of the carbon was $\varepsilon_{S, 0}=0.2$;

- the fluidized bed consists of $669 \mu \mathrm{m}$ alumina particles, fluidized by a $20 \% \mathrm{O}_{2} / \mathrm{N}_{2}$ gas mixture;

- $\mathrm{CO}_{2}$ is assumed to be the only primary combustion product $(a=1)$.

The results of a comparison of the numerical solutions of the ASPC model and the present composite solutions given by eq. (4.1) of Part I and eq. (4.5) with $\kappa=2$ and $\delta=1.3$, respectively, are discussed below.

Figures 11 and 12 show the particle overshoot temperature $\bar{\Psi}$ and the particle burn-out time $\Theta$, respectively, as a function of the initial particle diameter for two ambient temperatures. It is seen that for both $\bar{\Psi}$ and $\Theta$ there is reasonable to good agreement between the numerical results of Prins (1987) and the present composite solutions. It is remarkable that for small initial particle diameters, with $\phi_{0} \ll 1$, results do not compare very well. As the analytical solution for $\phi_{0} \ll 1$ is exact and rather simple, it is thought that the observed deviation might be due to some deficiency in the numerical calculation scheme of the ASPC model.

It is noted from the above results that the particle overshoot temperature as a function of the initial

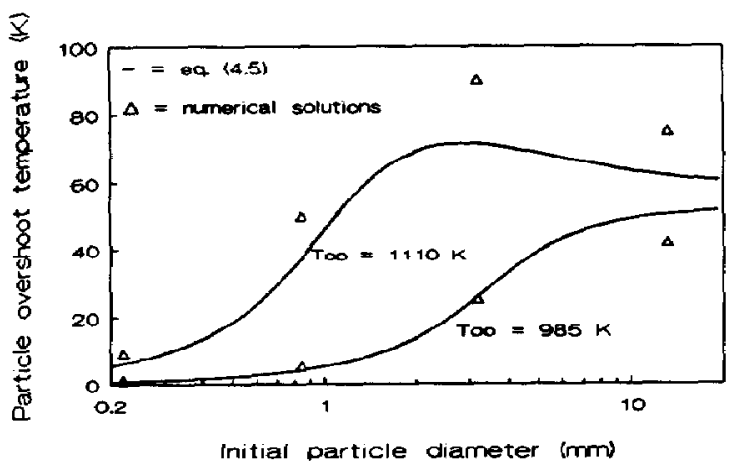

Fig. 11. Mean particle overshoot temperature as a function of the initial diameter of the carbon particle for $T_{\infty}=985$ and $1110 \mathrm{~K}$ : a comparison between numerical results of Prins (1987) and the present solutions of eq. (4.5).

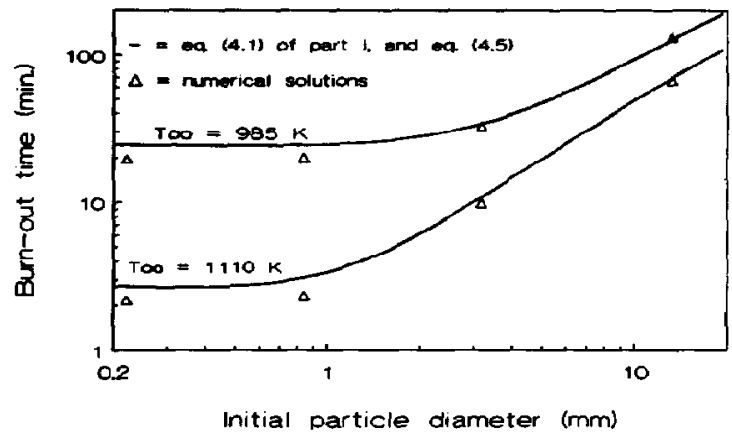

Fig. 12. Burn-out time $\left(X_{\infty}=0.9\right)$ as a function of the initial diameter of the carbon particle for $T_{\infty}=985$ and $1110 \mathrm{~K}$ a comparison between numerical results of Prins (1987) and the present solutions of eq. (4.1) of Part $I$.

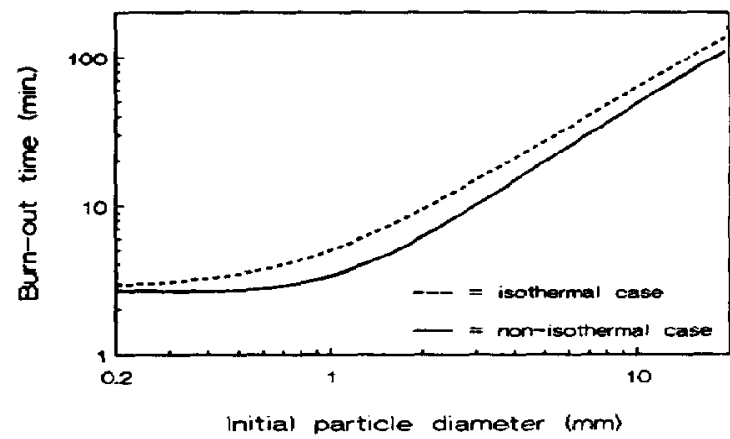

Fig. 13. Burn-out time $\left(X_{\infty}=0.9\right)$ as a function of the initial particle diameter for a non-isothermal and an isothermal situation. The parameter values are the same as used by Prins (1987) while $T_{\infty}=1110 \mathrm{~K}$.

particle diameter seems to pass through a maximum depending on the ambient temperature. For higher ambient temperatures the maximum overshoot temperature is higher and the maximum is reached for smaller particle diameters. Because of the maximum of the overshoot temperature the burn-out time may pass through a minimum.

Finally, Fig. 13 shows a comparison between an isothermal reaction and a non-isothermal reaction. For the non-isothermal reaction the calculation results, given in Fig. 12 for $T_{\infty}=1110 \mathrm{~K}$, are used again, while for the isothermal case the same conditions are used, except for the particle overshoot temperature, which is taken as equal to zero. It can be seen that there is only a slight difference between the isothermal and the non-isothermal solution, so, for this rather typical example of carbon combustion in a fluidized bed the extension to the non-isothermal case is seen to be of minor importance. For other conditions or for other reactants, the importance of the extension for the non-isothermal case could be much greater. The effects of the non-isothermal conversion can be assessed using the methods presented in this paper. 


\section{CONCLUDING REMARKS}

Complete analytical solutions for the non-linear conversion of a porous solid particle in a gas have been developed. The complete analytical solutions consist of a combination of two closed-form solutions asymptotically valid for $\phi_{0} \ll 1$ and $\phi_{0} \ll 1$. The solutions have been extended to non-isothermal cases, i.e. to include the effect of interaction between chemical reaction rate and particle overshoot temperature on particle conversion. The analytical solutions are shown to compare favourably with numerical simulation results.

In the solutions account has been taken of a variety of non-linear parameters, i.e. evolution of the intrinsic reaction surface area during conversion, evolution of pore diffusion during conversion, $m$ th-order reaction rate in gas reactant, critical particle porosity at the exterior surface, particle size decrease and particle overshoot temperature.

The analytical solutions presented in this paper are currently applied to model combustion of char particles in fluidized beds, the combustion of pulverized coal and the gasification of cokes. Furthermore, analytical methods similar to those of the present paper are used to describe $\mathrm{NO}_{x}$ formation and reduction inside and around a char particle, as well as sulfur capture of limestone particles. Some of these analytical single particle models are incorporated in a reactor model of a coal-fired fluidized-bed combustor to predict overall efficiencies and emissions (Brem, 1990). Numerical solutions of the above-mentioned particle models would result in an unacceptably long computational time and high costs of the reactor model. This demonstrates the importance of the presented solutions which provide an effective means for assessing the effect of particle conversion parameters on overall process behaviour. Insights thus obtained can be of direct use to the opcration and design of complete installations.

Acknowledgements-Financial support was received from TNO, the Netherlands Organization for Applied Scientific Research. The study was carried out within the framework of the Dutch National Research Program in Coal Technology, which is managed by NOVEM, the Netherlands Agency for Energy and Environent, and is financed by the Ministry of Economic Affairs.

\section{NOTATION}

a stoichiometric coefficient of the reaction

$A$ reduced reaction surface area $\left(A=A_{g} / A_{0}\right)$

$A_{g, 0} \quad$ reference value of reaction surface area

$A_{g} \quad$ reaction surface area per unit of volume

$B i_{h}$ Biot number for heat transfer $\left(B i_{h}=\right.$ $\left.B i_{h, c}+B i_{h, r}\right)$

$B i_{h, c}$ Biot number for convective heat transfer $\left(B i_{h, c}+R h \lambda_{e, 0}\right)$

$B i_{h, r}$ Biot number for randiant heat transfer $\left(B i_{k, r}=4 R \sigma \varepsilon_{r} T_{\infty}^{3} / \lambda_{e, 0}\right)$

\begin{tabular}{|c|c|}
\hline$B i_{m}$ & Biot number for mass transfer $\left(B i_{m}=R k_{d} /\right.$ \\
\hline$C$ & $\begin{array}{l}\text { reduced concentration of the gaseous re- } \\
\text { actant }\left(C=C_{A} / C_{A, \infty}\right)\end{array}$ \\
\hline$C_{A}$ & concentration of the gaseous reactant \\
\hline$c_{p}$ & specific heat of the particle \\
\hline$C_{S}$ & concentration of the solid reactant \\
\hline$D$ & reduced effective diffusivity $\left(D=D_{e} / D_{e, 0}\right)$ \\
\hline$D_{e}$ & effective diffusivity coefficient \\
\hline $\begin{array}{l}E_{0} \\
h\end{array}$ & $\begin{array}{l}\text { activation energy in Arrhenius expression } \\
\text { heat transfer coefficient in the external gas } \\
\text { layer }\end{array}$ \\
\hline$k_{d}$ & $\begin{array}{l}\text { mass transfer coefficient in the external gas } \\
\text { layer }\end{array}$ \\
\hline$\Delta H$ & heat of reaction \\
\hline$k_{s}$ & reaction rate constant \\
\hline Le & $\begin{array}{l}\text { Lewis number } \\
{\left[L e=a \lambda_{e, 0} C_{S, 0} /\left(\rho_{S} c_{p} C_{A, \infty} D_{e, 0}\right)\right]}\end{array}$ \\
\hline$m$ & $\begin{array}{l}\text { order of reaction with respect to the gaseous } \\
\text { reactant }\end{array}$ \\
\hline$n$ & $\begin{array}{l}\text { order of reaction with respect to the solid } \\
\text { reactant }\end{array}$ \\
\hline$r$ & radius co-ordinate \\
\hline $\boldsymbol{R}$ & initial particle radius, and also gas constant \\
\hline$S$ & $\begin{array}{l}\text { reduced concentration of the solid reactant } \\
\left(S=C_{S} / C_{S, 0}\right)\end{array}$ \\
\hline$S^{*}$ & $\begin{array}{l}\text { critical solid concentration at the outer sur- } \\
\text { face of the particle }\end{array}$ \\
\hline$t$ & time \\
\hline$T$ & particle temperature \\
\hline $\boldsymbol{u}$ & $\begin{array}{l}\text { reduced boundary layer velocity }(u= \\
\left.-\phi_{0} \partial \xi_{s} / \partial \Theta\right)\end{array}$ \\
\hline $\boldsymbol{X}$ & solid conversion \\
\hline$X_{\mathrm{as}}$ & $\begin{array}{l}\text { initial ash concentration of the particle, } \\
\text { based on mass }\end{array}$ \\
\hline
\end{tabular}

Greek letters

$\alpha \quad$ constant $\left[\alpha=1-\left(1-\varepsilon_{S .0}\right)\left(1-X_{\text {ash }}\right) \beta_{T} /\right.$ Le]

$\beta \quad$ exponent which characterizes the evolution of the effective diffusivity during conversion

$\beta_{T} \quad$ Prater number $\left[\beta_{T}=(-\Delta H) C_{, \infty} D_{a, a} /\right.$ $\left.\left(a \lambda_{e, 0} T_{\infty}\right)\right]$

$\gamma \quad$ Arrhenius number $\left(\gamma=E_{\mathrm{o}} / R T_{\infty}\right)$

$\delta$ fit parameter in compositc solution

$\varepsilon_{r} \quad$ emissivity of the external surface of the particle

$\varepsilon_{S} \quad$ particle porosity

$\Theta$ dimensionless time ( $\left.\Theta=k_{s} C_{A, \infty}^{m} A_{g, o} t / C_{s .0}\right)$

$\Theta_{c}$ time required for maximum conversion $X_{\infty}$

$\lambda \quad$ reduced heat conductivity $\left(\lambda=\lambda_{e} / \lambda_{e, 0}\right)$

$\lambda_{e} \quad$ effective heat conductivity

$\xi$

$\rho$

$\phi_{0}$

$\Psi$ reduced particle overshoot temperature

reduced radial co-ordinate $(\xi=r / R)$

particle density

Thiele modulus

$\left(\phi_{0}=R \sqrt{a k_{s} A_{g, 0} C_{A, \infty}^{m-1} / D_{e, 0}}\right)$ $\left(\Theta=T / T_{\infty}-1\right)$

$\Delta \Psi_{p}$ internal particle temperature gradient $\left(\Delta \Psi_{p}=\Psi-\Psi_{s}\right)$ 
Subscripts

$\begin{array}{ll}0 & \text { initial value } \\ 1 & \text { first region, } \phi_{0} \ll 1 \\ 2 & \text { second region, } \phi_{0} \gg 1 \\ \infty & \text { final situation, at ambient conditions } \\ A & \text { gas reactant } \\ \max & \text { maximum value } \\ S & \text { at external surface area of the particle } \\ S & \text { solid reactant }\end{array}$

Bliek, A., 1984, Mathematical modelling of a cocurrent fixed bed gasifier. PhD thesis, Twente University of Technology.

Brem, G., 1990, Mathematical modelling of coal combustion in an AFBC. PhD thesis, Twente University of Technology.
Froment, G. F. and Bischoff, K. B., 1979, Chemical Reactor Analysis and Design. John Wiley, New York.

Laurendeau, N. M., 1978, Heterogeneous kinetics of coal char gasification and combustion. Prog. Energy Combust. Sci. 4, 221-270.

Prins, W., 1987, Fluidized bed combustion of a single carbon particle. PhD thesis, Twente University of Technology.

Ranz, W. E. and Marshall, W. R., 1952, Evaporation from drops. Chem. Engng Prog. 48, 141-146.

Satterfield, C. N. and Sherwood, T. K., 1963, The Role of Diffusion in Catalysis. Addison-Wesley, Reading, MA.

Sotirchos, S. V. and Amundson, N. R., 1984, Diffusion and reaction in a char particle and in the surrounding gas phase. Two limiting models. Ind. Engng Chem. Fundam. 23, 180-191.

Timothy, L. D., Sarofim, A. F. and Beer, J. M., 1983 Characteristic of single particle coal combustion, in 19th Symp. (int.) Combust, pp. 1123-1130.

Wen, C. Y., 1968, Noncatalytic heterogeneous solid fluid reaction models. Ind. Engng Chem. Fundam. 60, 34-54. 